\title{
Uniform Moment Theory for Charged Particle Motion in Gases
}

\author{
Larry A. Viehland, ${ }^{1}$ William F. Siems ${ }^{2}$ \\ ${ }^{1}$ Science Department, Chatham University, Pittsburgh, PA 15232, USA \\ ${ }^{2}$ Department of Chemistry, Washington State University, Pullman, WA 99163, USA
}

\begin{abstract}
Moment equations for the motion of trace amounts of charged particles through dilute gases are developed from the Boltzmann equation. A new method for truncating the coupled moment equations is used to develop differential equations governing the moments in successive approximations. The first approximation equations are shown to agree completely with equations known to describe ion motion in drift-tube mass spectrometers, ion mobility spectrometers, ion traps, and collision-dominated ion cyclotron resonance experiments. Applications to differential mobility spectrometers and other devices are also described.
\end{abstract}

Key words: Ion mobility, Electron mobility, Gaseous ion transport, Boltzmann equation, Drifttube mass spectrometers, Ion mobility spectrometers, Ion traps, Ion cyclotron resonance, Differential mobility spectrometers

\section{Introduction}

$\mathrm{M}$ any mass spectrometers contain regions where significant neutral densities are present, such as when a collision chamber is used as a front-end device to either generate or accumulate ions or when there is an atmospheric pressure-vacuum interface with an electrospray ion source. These devices generally have a high enough pressure of a "buffer" gas that ion-neutral collisions must be taken into account in order to have a good understanding of the characteristics of the ions that are entering the low-pressure region, where the ions are sorted entirely by their masses. This understanding can, in favorable situations, lead to a second sorting of the ions, one based on collision cross sections. This is the situation, for example, in ion mobility spectrometry/mass spectrometry (IMS/MS) [1].

In 1975, Revercomb and Mason [2] summarized what was then known about the kinetic theory of the transport of trace amounts of ions through neutral gases under the influence of electrostatic fields. They directed their paper to analytical chemists interested in plasma chromatography or gaseous electrophoresis, old names for what is now called IMS. There have been many advances since then, and we have now

Correspondence to: Larry A.Viehland; e-mail: Viehland@chatham.edu reached the state where it is possible to start with accurate potential energy curves for atomic ion-atom interactions and calculate the transport coefficients with greater accuracy than they can be measured [3]. Similarly, the kinetic theory of electron transport is sufficiently advanced that electron transport coefficients can be accurately determined from information about the quantum-mechanical cross sections governing the electron-atom collisions [4]. Theory is in good shape, if not quite as advanced, for experiments involving timevarying electric fields or both electric and magnetic fields [4, 5]. Even the situation where one or both of the colliding particles is molecular has begun to be tractable [6]. The purpose of this paper is to present these developments in chemical physics and physical chemistry in a uniform way that will make them useful to analysts who need to understand the effects that ion-neutral collisions can have. We will present a closed system of differential equations governing the moments retained in the first of a series of approximations that provide progressively higher accuracy in describing the motion of trace amounts of ions through gases under the influence of arbitrary electric and magnetic fields. The assumption of trace ions means that we are considering swarm experiments and not plasmas [7] (i.e., ion-ion interactions are neglected).

This paper begins by showing how Newton's equations of motion are not sufficient for understanding ion motion in 
gases, primarily because ion-neutral collisions cannot be included in a fundamental way. We then introduce a uniform approach for solving the Boltzmann equation [8-10] that does properly account for ion or electron motion and collision with rare gas atoms, regardless of the experimental arrangements of external electric and/or magnetic fields. We will discuss gas mixtures and molecular ions or gases in later papers, and so here we allow the term "ions" to represent either atomic ions or electrons. It is impossible to present this work without some use of mathematics, but we keep this to a minimum by putting details into Appendices. Finally, we show how the first approximation of the present moment method for solving the Boltzmann equation leads to equations that are known to provide accurate descriptions of drift-tube mass spectrometers (DTMS), IMS, ion traps, and collision-dominated ion cyclotron resonance (ICR) experiments. Applications to differential mobility spectrometers (DMS) and other devices are also described.

\section{Elementary Theory}

Before beginning our considerations of kinetic theory, it is useful to consider a more primitive approach, based only on Newton's equation of motion for an ion with charge $q$, mass $m$, and velocity $\boldsymbol{v}$. If there are external electric and magnetic fields, $\boldsymbol{E}$ and $\boldsymbol{B}$, respectively, we assume that both are known functions of the time, $t$, and the ion position, $\boldsymbol{r}$. Then Newton's equation for the force, $\boldsymbol{F}=q(\boldsymbol{E}+\boldsymbol{v} \times \boldsymbol{B})$, on the ion may be written as a differential equation for the ion velocity,

$$
\frac{d}{d t} \boldsymbol{v}-\frac{q}{m}(\boldsymbol{E}+\boldsymbol{v} \times \boldsymbol{B})=0,
$$

where $x$ indicates the cross product of the vectors it connects. The problem with Equation (1) is that it does not take into account the effect of collisions that the ions have with atoms in the dilute gas through which they are dragged by $\boldsymbol{E}$ and $\boldsymbol{B}$. The usual way to compensate for this is to introduce an ad hoc damping term, $\check{\xi}$, to the left-hand side of Equation (1). The new quantity, $\xi$, has the units of inverse time, so it is referred to as the collision frequency. Unfortunately, there is no way within the context of Newton's equations to relate $\xi$ to the microscopic details (cross sections or interaction potentials) of the collisions. Neither is there any justification for the usual assumption that $\xi$ is constant. This is why we must turn to a kinetic theory based on the Boltzmann equation.

\section{Kinetic Theory}

The Boltzmann equation for a system composed of trace ions moving through a pure but dilute atomic gas [10] involves a general expression of forces on and velocity changes of the ions, as in Equation (1), and a collision operator that embodies resistance to the ionic motions due to collisions with the neutrals. This operator is linear if the ions are present in trace amounts and hence do not collide with one another. It is scalar, which means that it does not connect different components of any vector or tensor quantities (of arbitrary rank) upon which it operates. It is local in space, because the range of an ion-neutral interaction is negligible compared with the size of the apparatus. It is instantaneous in time, because the duration of a collision is negligible, compared with the time between collisions. Finally, we will work here with a collision operator in which the number densities of the ions and neutrals are explicitly indicated, rather than being included in the operator (as in [8-10]).

The Boltzmann equation incorporates the effect of both reactive and nonreactive ion-neutral collisions, and accounts therefore for nonconservation of ions. We will restrict our attention to the most common situation, where the reactive collisions are infrequent compared with the nonreactive ones. In this case the collision operator becomes a linear combination of a nonreactive operator, $J$, and a reactive operator, $J_{R}$; the complete expressions for these operators are given in [11]. The Boltzmann equation then has the form

$$
\begin{aligned}
& \left(\frac{\partial}{\partial t}+\boldsymbol{v} \cdot \nabla+\frac{q}{m}[\boldsymbol{E}+\boldsymbol{v} \times \boldsymbol{B}] \cdot \nabla_{\boldsymbol{v}}\right) f(\boldsymbol{r}, \boldsymbol{v}, t) \\
& \quad=N\left(J+J_{R}\right) f(\boldsymbol{r}, \boldsymbol{v}, t)
\end{aligned}
$$

where the unknown function, $f(\boldsymbol{r}, \boldsymbol{v}, t)$, is the ion velocity distribution function from which any measurable property can be determined by integration [10]. Here, $\boldsymbol{\nabla}$ is the spatial gradient vector, $\nabla_{v}$ is the velocity gradient vector, and $N$ is the number density of the neutrals.

Since $f(\boldsymbol{r}, \boldsymbol{v}, t)$ cannot be expressed in closed form for general circumstances, we often must content ourselves with determination of a limited set of velocity "moments", average values of certain specific functions of velocity. Although the correct value of $f(\boldsymbol{r}, \boldsymbol{v}, t)$ is more closely approximated as the number of known moments increases, it is inefficient to approximately determine $f(\boldsymbol{r}, \boldsymbol{v}, t)$ and then integrate to determine the few moments (average ion velocity and energy, for example) that can be compared with experiment. Instead, one generally attempts to solve directly for the moments.

In 1982, Weinert [12] developed a generalized moment method for treating the Boltzmann equation. He allowed the various species present to have arbitrary number densities, average velocities, and temperatures. This led him to a complicated "algebraization of the kinetic equation" that is related to Grad's 13-moment method [13]. Although Weinert made connections between his moment theory and the two-temperature theory of trace ion motion under the influence of electrostatic fields [14, 15], to the best of our knowledge it has not been used in other situations. It seems preferable to us to assume from the beginning that we are interested only in trace amounts of ions moving through 
dilute gases and thus develop an equivalent theory that contains less mathematics. In this regard, the present work is more like the moment theories given in chapter 5 of [10] and in chapter 6 of [16], except that the present moment theory is capable of handling time- and space-dependent electric and magnetic fields. It therefore shows some similarity to the recent work by Ender et al. [17], although the present work involves both a gas temperature and an ion temperature (the latter allows the trace amount of ions to have a large amount of energy due to strong external fields); the "standard moment method" developed in [17] was based on a single temperature for both species.

In order to determine equations that directly govern velocity moments for trace amounts of ions in dilute gases, we multiply Equation (2) from the left by any function, $\psi(v)$, of $v$ alone, and then integrate over all velocities. We get five terms that can be expressed in terms of general moments (averages over $\boldsymbol{v}$ ) defined by the relation,

$$
<\psi>=\frac{1}{n(\boldsymbol{r}, t)} \int \psi(\boldsymbol{v}) f(\boldsymbol{r}, \boldsymbol{v}, t) d \boldsymbol{v} .
$$

This definition reflects (but is not limited to) the most common situation, where the other moments of $f(\boldsymbol{r}, \boldsymbol{v}, t)$ vary much less rapidly with $\boldsymbol{r}$ and $t$ than does the ion number density,

$$
n(\boldsymbol{r}, t)=\int f(\boldsymbol{r}, \boldsymbol{v}, t) d \boldsymbol{v} .
$$

This is why the dependences of $\langle\psi>$ upon $\boldsymbol{r}$ and $t$ have been left implicit.

Two of the terms obtained from the Boltzmann equation are easy to write in terms of Equation (3), because the time derivative and $\nabla$ are independent of velocity and can be taken out of the integrals. A third term involves $\nabla_{v}$, but it can be written in terms of Equation (3) after integration by parts. The final terms involve $J$ and $J_{R}$, but they can also be written in this way if we use the inverse collision property of the collision operator [18]. Putting all of the pieces together gives the general equation for any moment,

$\frac{\partial}{\partial t} n(\boldsymbol{r}, t)<\psi>+\nabla \cdot n(\boldsymbol{r}, t)<\psi \boldsymbol{v}>-\frac{q}{m} n(\boldsymbol{r}, t)<[\boldsymbol{E}+\boldsymbol{v} \times \boldsymbol{B}] \cdot \nabla_{\boldsymbol{v}} \psi>$
$+n(\boldsymbol{r}, t) N<J \psi>+n(\boldsymbol{r}, t) N<J_{R} \psi=0$

where the center dot indicates a scalar product of the two vectors that it connects. This equation is equivalent to Maxwell's equation of change of 1867 [19], an alternative approach to the Boltzmann equation for the description of the transport properties of gases. In the absence of reactions, it is identical to the equation obtained by Kihara [20], although his derivation required that the collision operator be symmetric and positive definite. A derivation that does not include this requirement was given previously [14, 16] using essentially the procedure just described. Note, however, that we are neglecting any explicit effects of boundaries on the solution of Equation (5); these must be added later, when solutions of Equation (5) are attempted for particular experimental arrangements with initial and/or boundary conditions.

If we set $\psi(v)$ equal to 1, Equation (5) simplifies greatly, because a constant is one of the so-called collision invariants (i.e., it is an eigenfunction of $J$ whose corresponding eigenvalue is zero in this case, because of conservation of mass). We get the rate equation of continuity,

$$
\frac{\partial}{\partial t} n(\boldsymbol{r}, t)+\nabla \cdot n(\boldsymbol{r}, t)<\boldsymbol{v}>=-k N n(\boldsymbol{r}, t) .
$$

Here $k=<J_{R}>$ is the second-order reaction rate coefficient (since two particles are colliding). When there are no spatial gradients, Equation (6) reduces to the rate equation familiar to chemists. When there are no chemical reactions, Equation (6) reduces to the equation of continuity familiar to physicists.

Before Equation (6) can be solved for the ion number density, it is necessary to know the dependence of the moment of the average ion velocity, $\langle\boldsymbol{v}\rangle$, upon space and time. This is a familiar problem [7] in moment methods for solving Equation (5), since the moment equation for a simple quantity involves one or more moments of more complicated functions. Thus, the equation for $<\boldsymbol{v}>$, called the momentum-balance equation [16], involves the tensor $\langle\boldsymbol{v} \boldsymbol{v}>$ that is related to the temperature tensor. Similarly, the equation for $\left\langle\boldsymbol{v} \boldsymbol{v}>\right.$ involves the vector $\left\langle v^{2} \boldsymbol{v}\right\rangle$ that is related to the heat flux vector [16]. Deciding how to truncate this sequence by means of some closure or other approximation $[21,22]$ determines the type of "fluid equation" one has.

It is possible to systematically close the moment equations by working with an alternative to Equation (5). This procedure starts by using Equation (6) to replace the time derivative of $n(\boldsymbol{r}, t)$ in Equation (5) by spatial gradients. After dividing by $n(r, t)$ and making minor rearrangements, we get

$\frac{\partial}{\partial t}<\psi>-\frac{q}{m}<[\boldsymbol{E}+\boldsymbol{v} \times \boldsymbol{B}] \cdot \nabla_{\boldsymbol{v}} \psi>+N<J \psi>=R(\psi)$,

where

$$
\begin{aligned}
R(\psi)= & {[\langle\psi\rangle\langle\boldsymbol{v}\rangle-\langle\psi \boldsymbol{v}\rangle] \cdot \nabla \ln n(\boldsymbol{r}, t)+[\langle\psi\rangle \nabla \cdot\langle\boldsymbol{v}\rangle-\boldsymbol{\nabla} \cdot\langle\boldsymbol{v} \psi\rangle] } \\
& +k N\langle\psi\rangle-N<J_{R} \psi>.
\end{aligned}
$$

It should be noted that Equation (7) is exact for the regime where there are no initial or boundary conditions to consider and when chemical reactions are infrequent, by which we mean that it has the same level of mathematical rigor in this regime as the Boltzmann equation, Equation (2), or the original moment equation, Equation (5), from which it was derived.

The first term in $R(\psi)$ involves $\nabla \ln n(\boldsymbol{r}, t)$, which is the ratio of the gradient of the ion number density to $n(\boldsymbol{r}, t)$ itself. Not only is this ratio small in most situations, in the experiments we are interested in it is many times smaller yet when compared with $N$. This term also involves another small quantity, the difference between the product of two 
average quantities and the average of their product. Hence, we will ignore this term in the first of our series of successive approximation. This has one important consequence: the ion diffusion coefficients will not be treated until the second approximation is considered in a subsequent paper.

The second term in $R(\psi)$ is ordinarily neglected because spatial gradients are ignored [10] or it is approximated following careful consideration [16] of the heat flux vector, the temperature tensor and the like. This is where we will deviate most significantly from standard approaches: as described below, we will neglect this term in first approximation and treat it in subsequent steps in the hierarchy of systematic approximations.

The other terms on the right-hand side of Equation (8) incorporate the effects upon the transport properties of chemical reactions that lead to non-conservation of ions. Neglect of these terms is justified when such reactions are slow enough that $k\langle\psi\rangle$ and $\left\langle J_{R} \psi\right\rangle$ are small compared with the nonreactive terms in Equation (7). Although it is possible to treat situations with fast reactions [16], we will leave this for later work and from here on assume that $k\langle\psi\rangle$ and $<J_{R} \psi>$ can be neglected in first approximation.

Our method of solving Equation (7) is to assume in first approximation that $R(\psi)$ can be set to zero and that $\langle J \psi\rangle$ can be approximated based on the Maxwell model (see below). This results in a closed set of moment equations that can be solved for particular experimental situations. These equations can also be obtained by neglecting gradients in the ion number density and all other moments, by neglecting the effects of chemical reactions, and by making the same approximation to $\langle J \psi\rangle$. What makes the present approach different, however, is that in second approximation we keep not only one extra term in the expansion of $\langle J \psi\rangle$, but also the $R(\psi)$ terms in the equations that arise in first approximation. We end up with a larger, but still closed, set of coupled equations because we make the first approximation in the equations that arise for new moments. This procedure is iterated until the calculated results for the low-order moments of interest have converged to within a predetermined level of accuracy.

\section{Moment Equation for Velocity}

Putting $\psi(v)=\boldsymbol{v}$ into Equation (7) leads to the momentum balance equation,

$$
\frac{\partial}{\partial t}<\boldsymbol{v}>-\frac{q}{m}[\boldsymbol{E}+<\boldsymbol{v}>\times \boldsymbol{B}]+N<J \boldsymbol{v}>=R(\boldsymbol{v}) .
$$

We will consider this equation for several models.

\section{Maxwell Model}

We first consider the Maxwell model where the interaction potential between an ion and a neutral atom varies inversely as the fourth power of the separation. Since this is an excellent model for the long-range force between an atomic ion and a polarizable atom, it has been given considerable attention over the years [10]. In spherical polar coordinates in the lab frame of reference, the eigenfunctions of the Boltzmann collision operator for this model are Burnett functions $[10,15]$ of the dimensional ion velocity, v. For extensions beyond this model, it is better to use Burnett functions of a dimensionless ion velocity,

$$
\psi_{l, m}^{(r)}(\boldsymbol{v})=W^{l} S_{l+\frac{1}{2}}^{r}\left(W^{2}\right) Y_{l}^{|m|}(\theta, \phi) .
$$

Here the $Y_{l}^{|m|}(\theta, \phi)$ are the spherical harmonics of angles chosen with respect to the space-fixed axis, $z$, the $S_{l+\frac{1}{2}}^{r}\left(W^{2}\right)$ are Sonine (generalized Laguerre) polynomials of the squared magnitude of the vector

$$
\boldsymbol{W}=\left(\frac{m}{2 k_{B} T_{i}}\right)^{\frac{1}{2}} \boldsymbol{v},
$$

and $k_{B}$ is Boltzmann's constant. The ion temperature, $T_{i}$, is related to the average ion energy by the equation,

$$
\frac{3}{2} k_{B} T_{i}=<\frac{1}{2} m v^{2}>\text {. }
$$

Therefore, $T_{i}$ is an implicit function of $\boldsymbol{r}$ and $t$; its determination in terms of experimental parameters and microscopic quantities will be discussed in more detail below.

The Burnett functions are orthogonal with respect to the weighting function $\exp \left(-W^{2}\right)$. This implies that a good approximation to the ion velocity distribution function is the Maxwellian,

$$
f(\boldsymbol{r}, \boldsymbol{v}, t)=n(\boldsymbol{r}, t)\left(\frac{m}{2 k_{B} T_{i}}\right)^{\frac{3}{2}} \exp \left(-\frac{m v^{2}}{2 k_{B} T_{i}}\right) .
$$

It also implies that the nonreactive collision term in Equation (7) can be written for the Maxwell model as [14]

$$
<J \boldsymbol{v}>=a_{0,0}(1)<\boldsymbol{v}>,
$$

where the matrix elements designated as $a_{r, s}(l)$ must be diagonal in the $l$ index and independent of the $m$ index in the Burnett functions because $J$ is a spherical operator.

It has been shown [14] that for a pure neutral gas,

$$
a_{0,0}(1)=\frac{8}{3} \frac{M}{m+M}\left(\frac{2 k_{B} T_{e f f}}{\pi \mu}\right)^{1 / 2} \Omega^{(1,1)}\left(T_{e f f}\right)
$$

where $M$ is the neutral mass and

$$
\mu=\frac{m M}{m+M}
$$

is the ion-neutral reduced mass. The momentum-transfer collision integral, $\Omega^{(1,1)}\left(T_{\text {eff }}\right)$, is a weighted average of the 
energy-dependent, momentum-transfer cross section; it is a special case of the general equation [10]

$$
\Omega^{(l, s)}\left(T_{\text {eff }}\right)=\left[(s+1) !(k T)^{s+2}\right]^{-1} \int_{0}^{\infty} Q^{(l)}(\varepsilon) \exp \left(-\frac{\varepsilon}{k T_{e f f}}\right) \varepsilon^{s+1} d \varepsilon
$$

where the classical-mechanical expression for the transport cross section is given in terms of the impact parameter, $b$, and the scattering angle, $\theta(b, \varepsilon)$, by the expression

$$
Q^{(l)}(\varepsilon)=2 \pi\left[1-\frac{1+(-1)^{l^{-1}}}{2(l+1)}\right]^{-1} \int_{0}^{\infty}\left[1-\cos ^{l}(\theta(b, \varepsilon))\right] b d b .
$$

(Quantum expressions for the transport cross sections are given [10] in terms of the differences in phase shifts.) We note that the superscripts attached to a collision integral are not directly related to the indices of the matrix elements, $a_{r, s}(l)$.

The relationship between the scattering angle and the ionneutral interaction potential, $V(r)$, as a function of separation, $r$, is given by the equation $[10,14]$

$$
\theta(b, \varepsilon)=\pi-2 b \int_{r_{0}}^{\infty}\left[1-\frac{b^{2}}{r^{2}}-\frac{V(r)}{\varepsilon}\right]^{-\frac{1}{2}} \frac{d r}{r^{2}} .
$$

where the distance of closest approach, $r_{0}$, is the largest positive real root of the bracketed term. Therefore, the equations above provide a microscopic definition of the collision frequency for momentum-transfer,

$$
\xi\left(T_{e f f}\right)=N a_{0,0}(1)=\frac{8 N}{3} \frac{M}{m+M}\left(\frac{2 k_{B} T_{e f f}}{\pi \mu}\right)^{\frac{1}{2}} \Omega^{(1,1)}\left(T_{e f f}\right) .
$$

However, the quantity $T_{\text {eff }}{ }^{\frac{1}{2}} \Omega^{(1,1)}\left(T_{\text {eff }}\right)$ is constant for the Maxwell model, so the collision frequency for momentum transfer is constant for this model (and may be written simply as $\xi$ ).

In Equations (15-20), the effective temperature is

$$
T_{\text {eff }}=\frac{m T+M T_{i}}{m+M},
$$

where $T$ is the temperature of the neutral gas. Since $T$ and $T_{i}$ characterize the average energies in the laboratory frame for the neutral atoms and ions, respectively, $T_{\text {eff }}$ characterizes the average collision energy of a neutral and an ion in the center-of-mass frame. Like $<\boldsymbol{v}>$ and $T_{i}$, however, $T_{\text {eff }}$ is not at this point expressed in terms of experimental parameters and microscopic quantities.
In summary, the moment equation for the average ion velocity, when the Maxwell model is valid, is

$$
\frac{\partial}{\partial t}<\boldsymbol{v}>-\frac{q}{m}(\boldsymbol{E}+<\boldsymbol{v}>\times \boldsymbol{B})+\xi<\boldsymbol{v}>=R(\boldsymbol{v}) .
$$

This equation is similar to the ad hoc modification of Equation (1) to account for collisions, but only when we make the first approximation that the right-hand side can be neglected; even then, there is a difference in that the present equation applies to the average velocity of a swarm of ions and not to the behavior of a single ion in the swarm. Another word of caution is in order. The ioninduced dipole potential that justifies the Maxwell model is an accurate representation of the true ion-neutral interaction potential only at extremely large inter-nuclear separations. This means that the usual practice [10] of calculating the collision frequency or "polarization mobility" from the reduced mass and the value of the electric polarizability of the neutral is accurate only at extremely low temperatures, usually below $1 \mathrm{~K}$.

\section{Two-Temperature Model}

In 1975, it was recognized [14] that $T_{i}$ might be more important than it appears from the discussion of the Maxwell model. Since the experiments of interest here involve trace amounts of ions moving through dilute gases, the average energy of the ions can be substantially higher (due to the influence of strong electric fields) than thermal energy. Considerable success was obtained for experiments with cylindrical symmetry by using the Burnett functions with the ion temperature, $T_{i}$, (rather than the gas temperature, $T$, as had been used in previous approaches [10]) to expand the ion velocity distribution function. This leads [23] to a generalization of Equation (22),

$$
\begin{aligned}
\frac{\partial}{\partial t} & <\boldsymbol{v}>-\frac{q}{m}(\boldsymbol{E}+<\boldsymbol{v}>\times \boldsymbol{B})+\xi\left(T_{e f f}\right)<\boldsymbol{v}> \\
& =R(\boldsymbol{v})-\left[N<J \boldsymbol{v}>-\xi\left(T_{e f f}\right)<\boldsymbol{v}>\right],
\end{aligned}
$$

The additional terms on the right-hand side arise because $v$ is not an eigenfunction of $J$ for general ion-neutral interactions; since these terms will be non-zero only in second and higher approximation, we leave to a subsequent paper the task of evaluating them.

Equation (23) must be solved in order to determine $\langle\boldsymbol{v}\rangle$ in terms of the experimental parameters and microscopic properties. Even in first approximation, however, this cannot yet be attempted, because the collision frequency is a function of $T_{\text {eff }}$ through Equations (20-22). Hence, we will need to develop a moment equation for the effective temperature in the next section. 


\section{Rigid-Sphere Model}

For classical rigid-spheres of diameter $d$, the momentumtransfer cross section and the momentum-transfer collision integral both have the value $\pi d^{2}$. Hence, from Equation (20), the collision frequency for momentum-transfer for rigid spheres is

$$
\xi\left(T_{e f f}\right)=\frac{8 N}{3} \frac{M}{m+M}\left(\frac{2 k_{B} T_{e f f}}{\pi \mu}\right)^{\frac{1}{2}} \pi d^{2} .
$$

Note that for ion-neutral collisions in experiments of the kind of interest here, $d$ must be taken as the sum of the radii of the ion and neutral. Unfortunately, real ion-neutral interaction potentials are not as strongly repulsive at short separations as they are for rigid-sphere interactions. This means that even a well-chosen value of $d$ will represent experimental results only over small ranges of $T$ and $\frac{E}{N}$, a point that is often overlooked in modeling ion mobility experiments.

\section{Multi-Temperature Model}

In order to treat accurately situations that are intrinsically anisotropic, it is necessary to go beyond the two-temperature theory. The most straightforward extension for experiments with cylindrical symmetry is the three-temperature theory $[24,25]$ involving $T$ and average ion temperatures parallel and perpendicular to the cylindrical axis. A more general extension is the multi-temperature theory [23] that involves $T$ and three ion temperatures, each characterizing ion motion along one of the three mutually perpendicular axes in the apparatus. The multi-temperature theory is discussed in Appendix A.

\section{Moment Equations for Effective Temperature}

\section{Maxwell Model}

For the Maxwell model, where $\xi$ is constant, moment equations for the effective temperature are unnecessary. However, we can obtain such equations for this model by inserting the function, $\psi(v)=\frac{1}{2} m v^{2}$ into Equation (7). Although it is shown in Appendix A that the magnetic field affects components of the energy in the various directions, it is easy to show that the term involving $\boldsymbol{B}$ must vanish in this version of Equation (7). Thus, the magnetic field does not directly affect the total ion kinetic energy and we obtain the result

$$
\frac{\partial}{\partial t}\left(\frac{3}{2} k_{B} T_{i}\right)-q \boldsymbol{E} \cdot<\boldsymbol{v}>+<J\left(\frac{1}{2} m v^{2}\right)>=R\left(\frac{1}{2} m v^{2}\right),
$$

where we have made use of Equation (12). It is shown in Appendix B that, when the Maxwell model applies, Equation (25) becomes

$\frac{\partial}{\partial t}\left(\frac{3}{2} k_{B} T_{i}\right)-q \boldsymbol{E} \cdot\left\langle\boldsymbol{v}>+\frac{2 m}{m+M} \xi\left[\frac{3}{2} k_{B}\left(T_{i}-T\right)\right]=R\left(\frac{1}{2} m v^{2}\right)\right.$.

This is the equation that must be solved to determine the ion temperature in terms of experimental parameters and microscopic properties. Alternately, we can use Equation (21) to replace the ion temperature by the effective temperature, getting

$$
\frac{\partial}{\partial t}\left(\frac{3}{2} k_{B} T_{e f f}\right)-\frac{q \mu}{m} \boldsymbol{E} \cdot<\boldsymbol{v}>+\frac{2 \mu}{M} \xi\left[\frac{3}{2} k_{B}\left(T_{e f f}-T\right)\right]=\frac{\mu}{m} R\left(\frac{1}{2} m v^{2}\right)
$$

as the equation that must be solved to determine the effective temperature in terms of experimental parameters and microscopic properties.

\section{Two-Temperature Model}

As was the case for the moment equation for velocity, we can extend the Maxwell model result to apply to any ionneutral interaction potential within the two-temperature treatment of the Boltzmann equation. Thus Equation (27) becomes

$$
\begin{gathered}
\frac{\partial}{\partial t}\left(\frac{3}{2} k_{B} T_{\text {eff }}\right)-\frac{q \mu}{m} \boldsymbol{E} \cdot<\boldsymbol{v}>+\frac{2 \mu}{M} \xi\left(T_{\text {eff }}\right)\left[\frac{3}{2} k_{B}\left(T_{\text {eff }}-T\right)\right]=\frac{\mu}{m} R\left(\frac{1}{2} m v^{2}\right) \\
-\left\{\mathrm{N}<J\left(\frac{1}{2} m v^{2}\right)>-\frac{2 \mu}{M} \xi\left(T_{\text {eff }}\right)\left[\frac{3}{2} k_{B}\left(T_{\text {eff }}-T\right)\right]\right\} .
\end{gathered}
$$

This equation was given in this form in 2005 [23]. As with Equation (23), all of the terms on the right-hand side will be neglected in the first of our series of successive approximations.

\section{Rigid-Sphere Model}

For this model, the two-temperature moment equation for velocity, Equation (23), is valid, so long as the collision frequency for momentum transfer is given by Equation (24). Similarly, the moment equation for the effective temperature given by Equation (28) applies to this model when Equation (24) is used.

\section{Multi-Temperature Model}

The moment equations for the effective temperatures in the multi-temperature model are given in Appendix A.

\section{Drift-Tube Mass Spectrometers}

In the remainder of this paper we will restrict our attention to the first approximation, where we ignore the right-hand sides 


\section{A. Viehland and W. F. Siems: Charged Particle Motion In Gases}

of each of the moment equations developed above. Drift-tube mass spectrometers (DTMS) are defined here, following the physics literature, as instruments that are cylindrically-symmetric along the $z$ axis and in which there is a (possibly strong) electrostatic field along $z$, but no magnetic fields. The ion detector is interfaced with a mass spectrometer in order to be certain that the observed ions are the ones of interest. Often, a DTMS also has a mass spectrometer at the entrance to the drift tube, to keep out all ions except the one of interest. Special experimental techniques [10] are used in order to eliminate socalled end effects and the effects of chemical reactions, and to work at low pressures (100 Pa or less). This produces data [26] valid over a wide range of $E / N$, usually from 1 to $100 \mathrm{Td}$ or higher $\left(1 \mathrm{Td}=10^{-21} \mathrm{Vm}^{2}\right)$.

Measurements in DTMS are made at steady state, so we can drop the time derivatives in the moment equations. On the average, the ions move only along the $z$ axis, and their average speed along this direction is usually written as $v_{d}$. In such an apparatus, the first approximation to Equation (23) is

$$
v_{d}=\frac{q E}{m \xi\left(T_{e f f}\right)} .
$$

Similarly, the first-approximation to Equation (28) is

$$
\frac{2}{M} \xi\left(T_{e f f}\right)\left[\frac{3}{2} k_{B}\left(T_{e f f}-T\right)\right]=\frac{q}{m} E v_{d} .
$$

The ion mobility, $K$, is defined by the equation $v_{d}=K E$, while the standard (or reduced) mobility, $K_{0}$, is defined in terms of Loschmidt's constant, $N_{0}$, as $N_{0} K_{0}=N K$. Combining these equations with Equation (20) gives

$$
K_{0}=\frac{3 q}{8 N_{0}}\left(\frac{\pi}{2 \mu k_{B} T_{e f f}}\right)^{\frac{1}{2}} \frac{1}{\Omega^{(1,1)}\left(T_{e f f}\right)} .
$$

To the best of our knowledge, Equation (31) was first obtained by Hershey [27], who did not use kinetic theory but assumed that the ions have a Maxwellian distribution characterized by an ion temperature, while the neutrals have a similar distribution characterized by the gas temperature. He then used momentum-balance and energy-balance to obtain this fundamental ion mobility equation. The first kinetic-theory derivation of Equation (31) was by Viehland and Mason [14].

Since the same collision frequency arises in both Equations (29) and (30), we can solve these equations for the effective temperature at steady state by eliminating $\xi\left(T_{\text {eff }}\right)$. This gives

$$
\frac{3}{2} k_{B} T_{e f f}=\frac{3}{2} k_{B} T+\frac{1}{2} M v_{d}^{2} .
$$

Equations (29) and (32) are the desired expressions (in first approximation) for the drift velocity and effective temperature in DTMS, in terms of experimental parameters and microscopic properties.
When combined with Equation (21), Equation (32) gives the Wannier formula [28],

$$
\frac{3}{2} k_{B} T_{i}=\frac{3}{2} k_{B} T+\frac{1}{2} m v_{d}^{2}+\frac{1}{2} M v_{d}^{2} .
$$

Note that Equation (33) shows that the ion energy in the laboratory frame consists of three pieces: thermal energy, kinetic energy that the ions have absorbed from the external field and are exhibiting as motion along the field axis, and kinetic energy that the ions have similarly absorbed but that has been transformed into random motion due to collisions with the neutrals.

There is no assumption about the type of ion-neutral interaction built into the present derivation of the Wannier formula. It follows here directly from the first approximation moment theory and a single assumption: the ion velocity distribution function is represented with reasonable accuracy as a Maxwellian distribution characterized by an ion temperature that may be much larger than the gas temperature. (The use of Burnett functions was also dictated by this assumption, since they are orthogonal with respect to such a Maxwellian distribution function.) In this way, the Wannier formula arises in essentially the same manner as Hershey [27] used to obtain Equation (31).

The combination of the fundamental ion mobility equation with the Wannier formula is known $[10,14,15]$ to give results accurate within $10 \%$. As an illustration, Figure 1 shows two sets of calculated values for the mobilities of $\mathrm{Cs}^{+}$and $\mathrm{I}^{-}$ions in $\mathrm{He}$ at $300 \mathrm{~K}$. The solid curves were calculated with a precision of $0.1 \%$ from ab initio potentials [26, 29, 30]; if the potentials are as accurate as expected, the calculated mobilities have an accuracy of

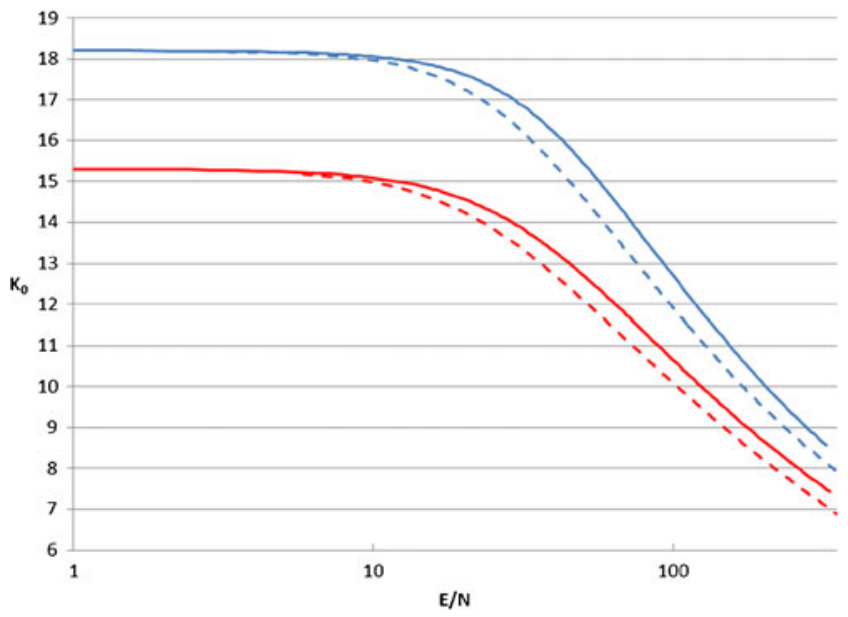

Figure 1. Reduced mobility, $K_{0}$ in $\mathrm{cm}^{2} \mathrm{Ns}$, as a function of the reduced field strength, $\frac{E}{N}$ in $\mathrm{Td}$, for $\mathrm{Cs}^{+}$ions (blue, upper curves) and $\mathrm{I}^{-}$ions (red, lower curves) in $\mathrm{He}$ at $300 \mathrm{~K}$. All values were calculated from the ab initio potentials $[29,30]$. The solid curves are expected to differ by no more than $0.1 \%$ from the experimental values, while the dashed curves represent values calculated using the present first-approximation to the two-temperature moment equations 
$0.1 \%$. The dashed curves in Figure 1 were calculated from Equations (31) and (32) using a newly-written Fortran program and the momentum-transfer cross sections obtained from the same interaction potential energy functions. The disagreement is no more than $5 \%$ over the entire range of $\frac{E}{N}$. It has been known since 1978 [15] that such disagreements are negligible at small $\frac{E}{N}$, and on the order of $10 \%$ at high $\frac{E}{N}$, for heavy ions moving in light gases at temperatures where thermal energy is above the well depth.

It is worthwhile at this point to note the similarities and differences between the momentum-transfer cross section and collision integral. They both have the dimensions of distance squared and (by means of the normalization factors included in the definition) they become identical in the special case of rigid spheres. However, for general interactions $\Omega^{(1,1)}\left(T_{e f f}\right)$ is a function of the effective temperature that is equivalent to the average collision energy, whereas $Q^{(1)}(\varepsilon)$ is a function of the relative kinetic energy, $\varepsilon$, for a single collision. As Figure 2 shows, these relationships are similar, but not identical, for general ionneutral interaction potentials. At very low energies, the results shown in Figure 2 are qualitatively similar; this reflects the fact that the dominant interaction is the ion-induced dipole interaction (i.e., the Maxwell model applies). It should be noted, however, that the graph is logarithmic, so the weight factor involved with the averaging to produce $\Omega^{(1,1)}\left(T_{\text {eff }}\right)$ results in a value that is a factor of 2-3 smaller than $Q^{(1)}(\varepsilon)$ for the Maxwell model.

At very high energies, $Q^{(1)}(\varepsilon)$ and $\Omega^{(1,1)}\left(T_{\text {eff }}\right)$ have similar behaviors in Figure 2 for each of the two ions, but again they are not identical. This indicates that the interaction potentials at very short separation are highly repulsive, but not as repulsive as rigid spheres. This comment is supported by Figure 1, which shows that the mobility at high $\frac{E}{N}$, while decreasing, does not fall off like

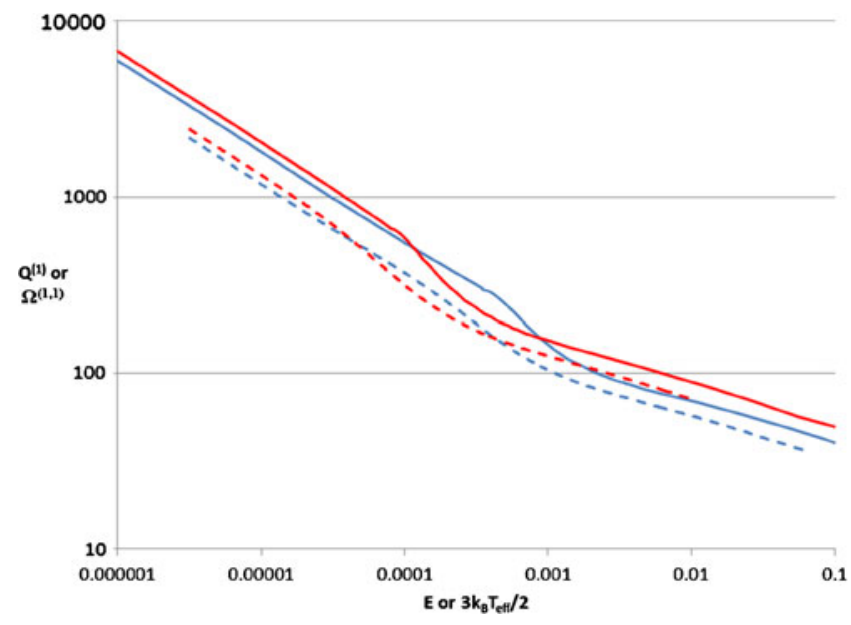

Figure 2. Momentum-transfer cross sections (solid curves), $Q^{(1)}$, as a function of the collision energy, $\varepsilon$, for a single ionneutral collision and momentum-transfer collision integrals (dashed curves), $\Omega^{(1,1)}$, as a function of the average collision energy, $\frac{3 k_{B} T_{e f f}}{2}$, for $\mathrm{Cs}^{+}$ions (blue, generally lower curves), and $\mathrm{I}^{-}$ions (red, generally higher curves) in He. Atomic units are used for all quantities the inverse-half power of $\frac{E}{N}$, as it would if the ion-helium potentials were like rigid spheres.

At intermediate energies, Figure 2 shows more structure in $Q^{(1)}(\varepsilon)$ than in $\Omega^{(1,1)}\left(T_{e f f}\right)$. Structure in the transport cross sections is common [31], if not universal, for atomic ion-atom interactions. It occurs at and somewhat above the maximum energy at which the interaction potential will allow the colliding particles to orbit indefinitely around one another. This structure does not carry over into the momentum-transfer collision integral due to the averaging over energies that is necessary to compute $\Omega^{(1,1)}\left(T_{e f f}\right)$. However, it does result in the changes in slope shown in Figure 2, which in turn carry over into a mobility maximum at $100 \mathrm{~K}$. The reason that such a maximum does not appear in Figure 1 is that thermal energy at room temperature is already higher than the energy at which the changes in slope occur in Figure 2. As a final comment, the dashed curves in Figure 3 are, except for constants, plots of the collision frequencies for momentum transfer, $\xi\left(T_{\text {eff }}\right)$.

\section{Ion Mobility Spectrometers}

In the chemistry literature, a DTMS operated at low values of $\frac{E}{N}$ (because the electric field is weak, the gas pressure is at or near atmospheric pressure, or both) is usually referred to as an IMS or an IMS/MS [1]. Here, we will use the term IMS to refer to a DTMS that is operated at such low values of $\frac{E}{N}$ that $T_{i}$ and $T_{\text {eff }}$ are essentially equal to $T$. An important experimental question is just how low this value must be. A criterion was put forth long ago [2], but it was based on theoretical quantities that can only be estimated. An alternative criterion was obtained [32] by requiring the last two terms in Equation (33) to be small. It is

$$
\frac{E}{N} \leq \frac{1}{5 N_{0} K_{0}}\left(\frac{3 k_{B} T}{m+M}\right)^{\frac{1}{2}}
$$

where the factor of 5 was introduced empirically. The key point is that only measurable properties are needed in order

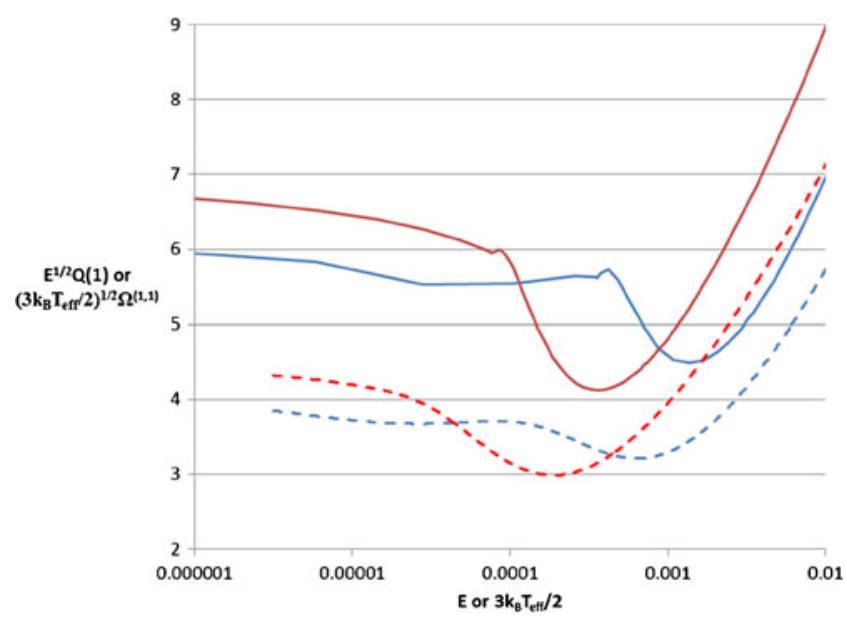

Figure 3. Same as Figure 2 but with $Q^{(1)}$ multiplied by the square root of $\varepsilon$ and $\Omega^{(1,1)}$ muliplied by the square root of $\frac{3 k_{B} T_{\text {eff }}}{2}$ 
to use this criterion. At $300 \mathrm{~K}$ and low $\frac{E}{N}, K_{0}$ is $18.20 \mathrm{~cm}^{2} /$ Vs for $\mathrm{Cs}^{+}$in $\mathrm{He}$ and $15.30 \mathrm{~cm}^{2} / \mathrm{Vs}$ for $\mathrm{I}^{-}$in He. Then Equation (34) predicts that the mobilities are constant below $0.956 \mathrm{Td}$ for ${ }^{133} \mathrm{Cs}^{+}$in $\mathrm{He}$ and below $1.16 \mathrm{Td}$ for ${ }^{127} \mathrm{I}^{-}$in $\mathrm{He}$. These conservative predictions are in agreement with the results shown in Figure 1.

When the criterion in Equation (34) is satisfied, Equation (31) reduces to

$$
K_{0}=\frac{3 q}{8 N_{0}}\left(\frac{\pi}{2 \mu k_{B} T}\right)^{\frac{1}{2}} \frac{1}{\Omega^{(1,1)}(T)} .
$$

This expression for the zero-field mobility was first obtained in 1905 by Langevin [33], and cited by Hassé and Cook in 1931 [34] and by Tyndall in 1938 [35]. The notation then in use was quite different, however, and Langevin's derivation involved the determination of the diffusion coefficient and then the use of the Nernst-Townsend-Einstein relation [10]. The first derivation through Equation (31) was given in 1939 by Hershey [27]. The first proper derivation, through a kinetic theory valid only at low fields, was given in 1953 by Kihara [20] and later cited by Mason and Schamp [36]. Since this history is rather convoluted, we recommend that Equation (35) not be given anyone's name but, instead, be referred to as the fundamental low-field ion mobility equation. This is parallel to calling Equation (31) the fundamental ion mobility equation.

Since the momentum-transfer collision integral is often unknown, it is customary in IMS/MS to assume that it has the value $\pi d^{2}$ and to make some physically-reasonable estimate for $d$. Since $\Omega^{(1,1)}(T)$ does not change as long as the apparatus is used at the same temperature, there is no reason not to do this. In terms of Figures 1, 2, 3, if we remain at the same average collision energy, it does not matter how we obtain an appropriate value for $\Omega^{(1,1)}(T)$. However, it must not be assumed that the collisions are so energetic that they are probing the short-range region of the ion-neutral interaction potential, where a rigid-sphere interaction might be justified microscopically. This is illustrated by the variations of $\Omega^{(1,1)}(T)$ shown in Figures 2 and 3. To illustrate this in another way, Figure 4 shows that the zerofield mobilities for $\mathrm{Cs}^{+}$and $\mathrm{I}^{-}$ions in Ar increase with $T$ up to $800 \mathrm{~K}$, which means that the collisions are still dominated by the long-range components of the interactions. Note also that Figure 4 shows that the polarization mobility, also called the Langevin mobility, is not achieved until the gas temperature is considerably below $1 \mathrm{~K}$. Finally, Figure 4 shows that the zero-field mobility can sometimes be a strong function of $T$; this variation must be carefully taken into account when comparing IMS/MS measurements made under different conditions [37].

\section{Ion Cyclotron Resonance}

In simplest form, ICR experiments involve a swarm of ions of very low density moving through a dilute neutral gas

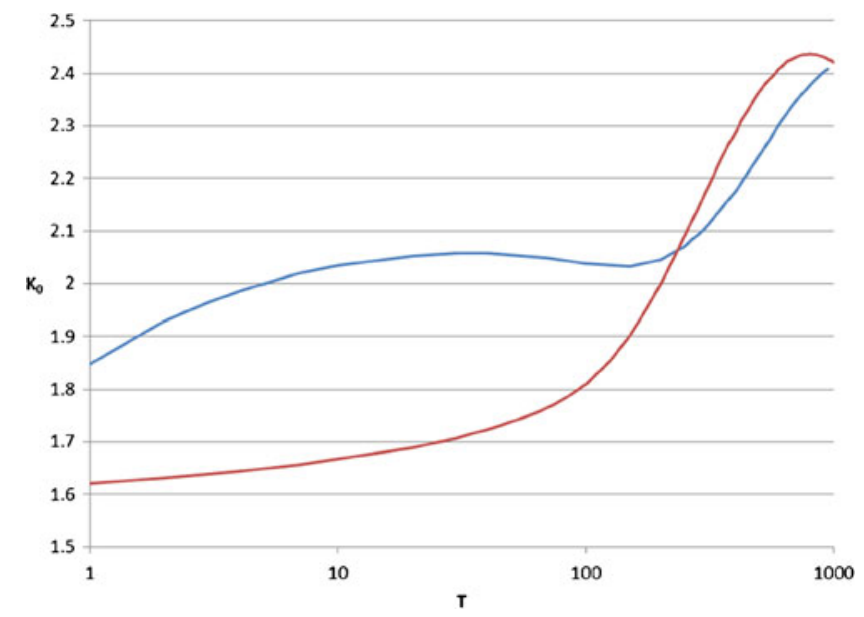

Figure 4. Zero-field mobility, $K_{0}^{(0)}$ in $\mathrm{cm}^{2} / \mathrm{Vs}$, of $\mathrm{Cs}^{+}$ions in Ar (blue, generally higher curve) and $\mathrm{I}^{-}$ions in $\mathrm{Ar}$ (red, generally lower curve), as a function of the gas temperature, $T$ in K. All values were calculated from the ab initio potentials $[29,30]$. The polarization limit is the value of $K_{0}^{(0)}$ that should be reached at $0 \mathrm{~K}$; the limits for these systems are nearly identical because the ion-Ar reduced masses are very similar

under the influence of a constant magnetic field and an oscillating electric field that is perpendicular to $\boldsymbol{B}$. If we let the magnetic field define the $z$ axis, this field causes the ions to rotate in the $x-y$ plane with a sharp cyclotron frequency, $\omega_{c}=\frac{q B}{m}$, independent of $\boldsymbol{r}$ and $\boldsymbol{v}$. At low pressures, the application of an ac field perpendicular to $\boldsymbol{B}$ with frequency $\omega=\omega_{c}$ causes the ions to absorb energy, leading to a measurable ICR signal. At high pressures, a finite line width of the signal arises from ion-neutral collisions, so the measurements can provide information about nonreactive ion-neutral collisions.

In a more typical ICR experiment, the ions are drifted through the apparatus by inhomogeneous electric fields such that $E_{x}=E_{0} \cos (\omega t), E_{y}=E_{1}$, and $E_{z}=E_{2}$ where $E_{0}, E_{1}$, and $E_{2}$ are constants. Then setting the right-hand side of Equation (23) to zero gives the first-approximation equations:

$$
\begin{gathered}
{\left[\frac{d}{d t}+\xi\left(T_{e f f}\right)\right]<v_{x}>=\frac{q}{m} E_{0} \cos (\omega t)+\omega_{c}<v_{y}>,} \\
{\left[\frac{d}{d t}+\xi\left(T_{e f f}\right)\right]<v_{y}>=\frac{q}{m} E_{1}-\omega_{c}<v_{x}>,}
\end{gathered}
$$

and

$$
\left[\frac{d}{d t}+\xi\left(T_{e f f}\right)\right]<v_{z}>=\frac{q}{m} E_{2} .
$$

When the collision frequency is constant (the Maxwell model), these equations can be solved [38] by Fourier transforms and used to determine absorption line widths. 
For more general ion-neutral interactions, where $\xi\left(T_{e f f}\right)$ is not constant, we must add to the three differential equations above another equation obtained by setting the right-hand side of Equation (28) to zero. We obtain

$$
\begin{aligned}
\frac{d}{d t}\left(\frac{3}{2} k_{B} T_{e f f}\right) & =\frac{q \mu}{m}\left[E_{0} \cos (\omega t)<v_{x}>+E_{1}<v_{y}>+E_{2}<v_{z}>\right] \\
& -\frac{2 \mu}{M} \xi\left(T_{e f f}\right)\left[\frac{3}{2} k_{B}\left(T_{e f f}-T\right)\right] .
\end{aligned}
$$

The combined use of Equations (36)-(39) was demonstrated some time ago [39]. Because the focus of ICR research moved to collision-free rather than collisiondominated experiments, no recent work along these lines appears to have been carried out. If interest returns to collision-dominated ICR, it would be useful to formulate a multi-temperature theory of ICR and to extend both the twoand multi-temperature theories to describe Fourier-Transform ICR experiments.

\section{Differential Mobility Spectrometry}

Field-asymmetric ion mobility spectroscopy (FAIMS) and DMS experiments separate gas-phase ions at atmospheric pressure by combining low- and high- $\frac{E}{N}$ measurements of $K_{0}$. The principles of operation of such devices with parallel plate geometry were described by Buryakov et al. [40]. The use of concentric cylinders for FAIMS was advocated by Guevremont and Purves [41], in order to avoid difficulties in working with a rectangular asymmetric waveform [42].

Analysis of FAIMS and DMS experiments assumes that the transition between the low- and high- $E / N$ regimes occurs so rapidly that the data can be analyzed entirely in terms of different (constant) mobilities at low and high reduced fields. In principle, the first-approximation moment equations given here could be used to test quantitatively the validity of this assumption, but this would essentially be repeating a study that was undertaken long ago [43]. That study showed that a change in $\frac{E}{N}$ has an effect on the mobility over a much shorter period of time than it does on the ion energy; the new mobility differs by less than $1 \%$ from its final value after the approximate time $\tau=\frac{4 m N_{0} K_{0}}{q N}$. For $\mathrm{Cs}^{+}$ions in He with $T=300 \mathrm{~K}, N=N_{0}$, and the electric field so low that $K_{0}=18.20 \mathrm{~cm}^{2} / \mathrm{Vs}$, this equation gives $\tau \approx$ 10 ns. Consequently, it is safe to make this assumption in FAIMS and DMS, although it should be noted that working at sufficiently low gas densities could invalidate the assumption. In addition, using asymmetric waveforms with many different values of $\frac{E}{N}$ in order to obtain higher-order DMS separations [44] would cause the assumption to break down at values of $N$ closer to $N_{0}$.

It is easy to show [10] that the ion mobility must be an even function of $\frac{E}{N}$. Suppose the electric field originally defined the $+z$ axis but that we decide to change the coordinate system so that $+z$ becomes $-z$. Since nothing changes about the physical apparatus, this requires the electric field vector to change from
$\boldsymbol{E}$ to $-\boldsymbol{E}$ and the drift velocity vector to change from $\boldsymbol{v}_{d}$ to $-\boldsymbol{v}_{d}$. Since the mobility is the proportionality constant between these vectors, its value cannot change. In order for $K_{0}$ to remain unchanged when $\frac{E}{N}$ changes sign, it can only depend upon $\left(\frac{E}{N}\right)^{2}$. However, this argument does NOT mean that $K_{0}$ should be written as a series in powers of the square of $\frac{E}{N}$, even though that is ordinarily assumed in FAIMS and DMS work. Indeed, it was demonstrated long ago [45] that the radius of convergence for such an expansion is small; for $\mathrm{K}^{+}$ions in $\mathrm{He}$ and $\mathrm{Ne}$ at or near room temperature, only $\frac{E}{N}$ values up to $20 \mathrm{Td}$ can be obtained from a theory that assumes the field to be zero.

The success achieved with the first approximation for DTMS, as shown in Figure 1, means that this approximation can be used to find a better way of describing the change of the mobility as the field strength increases in FAIMS or DTMS. Equation (31) shows that there is essentially one independent variable $T_{\text {eff }}$, rather than the two ordinarily considered, $T$ and $\frac{E}{N}$; this was first noted in 1975 [14]. Since Equation (32) is equivalent to

$$
\frac{3}{2} k_{B} T_{\text {eff }}=\frac{3}{2} k_{B} T+\frac{1}{2} M N_{0}^{2}\left(\frac{E}{N}\right)^{2} K_{0}^{2},
$$

the first approximation to the effective temperature is an even function of $\frac{E}{N}$, like $K_{0}$. Note that Equation (40) allows $T_{\text {eff }}$ to be calculated from experimental quantities with the use of only simple algebra. To illustrate the value of this quantity, Figures 5 and 6 show the same data as Figure 1 and similar results for gas temperatures of 400 and $500 \mathrm{~K}$, but with the independent variables being $\left(\frac{E}{N}\right)^{2}$ and $T_{\text {eff }}$, respectively, rather than $\frac{E}{N}$. Note, in particular, that a straight line describing the low-field results in Figure 5 is a poorer representation of all of the data than is true for a similar straight line in Figure 6 (i.e., deviations set in at smaller values of the square of $\frac{E}{N}$ than of $\left.T_{\text {eff }}\right)$. This means

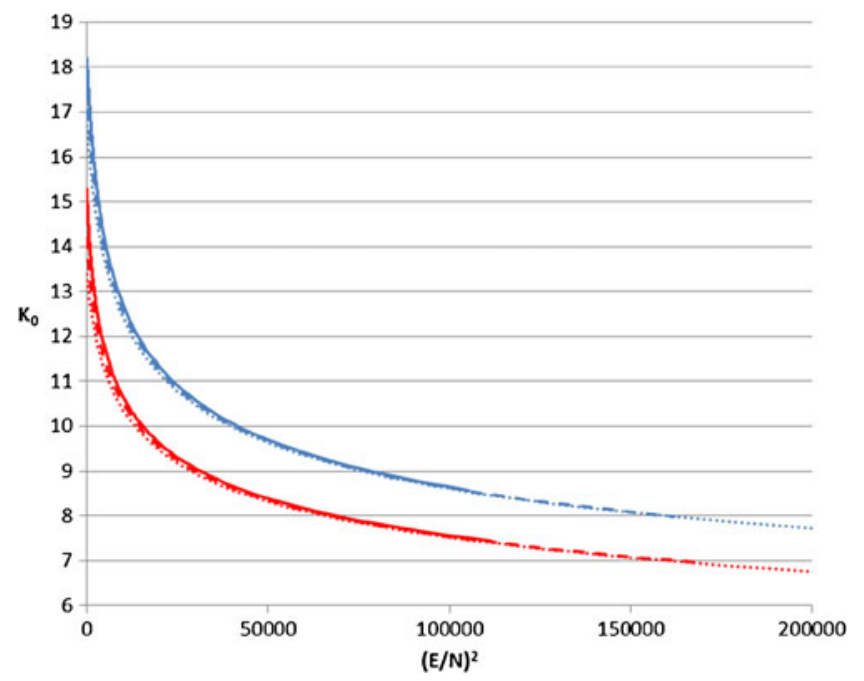

Figure 5. Reduced mobility, $K_{0}$ in $\mathrm{cm}^{2} \mathrm{Ns}$, of $\mathrm{Cs}^{+}$(blue, higher curves) and $\mathrm{I}^{-}$(red, lower curves) in $\mathrm{He}$ at $300 \mathrm{~K}$ (solid), $400 \mathrm{~K}$ (large dashes), and $500 \mathrm{~K}$ (dots), as a function of the square of $\frac{E}{N}$ in square $\mathrm{Td}$ 


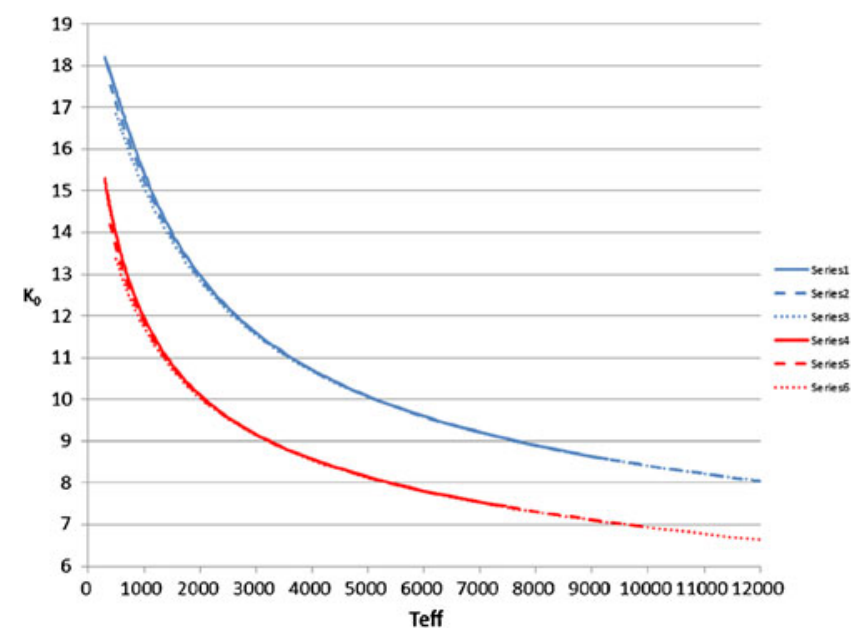

Figure 6. Same as Figure 5, as a function of $T_{\text {eff }}$ in $\mathrm{K}$

that the first two terms in a power series expansion in $\left(\frac{E}{N}\right)^{2}$ does not describe the mobility to as high values of $\frac{E}{N}$ as do the first two terms in the equivalent expansion in powers of $T_{\text {eff. }}$ This is true even if the results at the different temperatures are displaced so that their zero-field values match those at $300 \mathrm{~K}$.

\section{Ion Traps}

Because of the complicated geometrical shape in ion traps, the multi-temperature theory is generally preferable to the twotemperature theory for them. For an ideal quadrupole ion trap, the first approximation discussed in Appendix A leads to a system of six coupled, ordinary differential equations that can be solved by standard numerical methods [46].

A stretched or non-ideal quadrupole ion trap is cylindrically symmetric along the $z$ axis, but has a more complicated electric field than in an ideal quadrupole trap. Moreover, a time-dependent electric potential may be applied between the hemispherical end caps in a dipolar fashion. A multitemperature moment theory of non-ideal ion traps has already been presented [47]. Similar work for linear ion traps is in progress.

The results obtained by solving the first-approximation moment equations have been used [5] to make the first $a b$ initio calculations of ion-neutral reaction rate coefficients in ion traps. It is worthwhile to note also that the uniform way in which moment theory has led to well-established results for ion mobility and energy in DTMS, IMS/MS, ICR, and DMS lends considerable support to the expectation that these moment equations are accurate for ion traps.

\section{Other Apparatus}

Penning traps are devices for the storage of charged particles using a strong, homogeneous, axial, static magnetic field, and a spatially inhomogeneous, static, quadrupole electric field to confine the particles axially. To describe the motion of trace amounts of ions in such devices when collisions occur with a dilute, nonreactive background gas, we need the full set of first-approximation moment equations from the multi-temperature approach given in Appendix A. Although it is certainly feasible, no work has yet been published approaching Penning traps in this manner.

As noted above, the first approximation equations derived here can be obtained by other approximations to Equation (5). Accordingly, applications of such equations have been given by others for situations where reacting ion swarms are controlled by DC electric fields [48], harmonically varying AC electric fields [21], and crossed electric and magnetic fields $[4,49]$. We note, however, that our second approximation will be slightly different than obtained by other approximations for closing the moment equations, so further work will be required to determine the importance of such differences.

\section{Conclusions}

Starting from the Boltzmann equation, coupled differential equations have been developed for moments of the ion velocity distribution function in experiments involving trace amounts of atomic ions moving through dilute atomic gases. The first approximation of the two-temperature approach is best suited for experiments with cylindrical symmetry, for then the only moments that are involved are the three components of the average ion velocity, $\langle\boldsymbol{v}\rangle$, and the effective temperature, $T_{\text {eff }}$, which is equivalent to the average ion energy. These four quantities are governed by the vector equation

$$
\frac{d}{d t}<\boldsymbol{v}>-\frac{q}{m}(\boldsymbol{E}+<\boldsymbol{v}>\times \boldsymbol{B})+\xi\left(T_{\text {eff }}\right)<\boldsymbol{v}>=0
$$

and the scalar equation

$\frac{d}{d t}\left(\frac{3}{2} k_{B} T_{e f f}\right)-\frac{q \mu}{m} \boldsymbol{E} \cdot<\boldsymbol{v}>+\frac{2 \mu}{M} \xi\left(T_{\text {eff }}\right)\left[\frac{3}{2} k_{B}\left(T_{e f f}-T\right)\right]=0$,

where the collision frequency for momentum transfer is defined by Equation (20). Once the electric and magnetic fields, the gas temperature and number density, and the ionneutral interaction potential (equivalently, $\xi\left(T_{\text {eff }}\right)$ ) are given, it is a rather straightforward numerical task to solve this system of four coupled ordinary differential equations. This is in stark contrast to a previous claim [17] that a moment theory using two temperatures "gives rise to ten thousands of" moments; such large numbers of moments are required only if one is interested in the ion velocity distribution function itself.

The multi-temperature procedure in Appendix A is the approach best suited for situations that do not have cylindrical symmetry. In the first approximation of this approach, there are six quantities involved - the three components of the average ion velocity and the three components of the average ion energy along the Cartesian 
axes - and six coupled ordinary differential equations that must be solved.

Both theories discussed here use ion velocities in the laboratory frame, $\boldsymbol{v}$. It is possible to instead work in the frame of the ion swarm [16] by using "peculiar" velocities, $\boldsymbol{v}-\boldsymbol{v}_{\boldsymbol{d}}$, but there does not appear to be any advantage in doing this from the very beginning. If desired, one can convert the final results in the present theories into the other frame of reference with no special difficulty. This will be illustrated in a subsequent publication.

The first approximation equations have been applied here to several types of experiment. For DTMS, the results of the two-temperature approach have been shown to be exactly the same as those of the first approximation of the two-temperature kinetic theory that is known to be accurate within approximately $10 \%$ at all values of $T$ and $\frac{E}{N}$. For IMS, the present two-temperature results give the fundamental low-field ion mobility equation that follows from the fundamental ion mobility equation; both are known to be very accurate. The uniform nature of the theory presented here and the accuracy of the first approximations for DTMS, IMS, ICR, DMS, and ion traps, suggest that similar calculations for other experiments (some already published and others still underway) should also be accurate.

\section{Appendices}

\section{A. Multi-temperature Theory}

For the multi-temperature model in Cartesian coordinates, the Burnett functions in Equation (10) must be replaced by a product of three Hermite polynomials, one for each direction. The basis functions are

$$
\psi_{p, q, r}(\mathbf{v})=H_{p}\left(W_{x}\right) H_{q}\left(W_{y}\right) H_{r}\left(W_{z}\right),
$$

where

$$
W_{u}=\left(\frac{m}{2 k_{B} T_{u}}\right)^{\frac{1}{2}} v_{u}
$$

and $u=x, y, z$ labels a Cartesian axis. Then it is possible [23] to proceed as in the two-temperature model and obtain an extension of Equation (23),

$$
\begin{aligned}
& \frac{d}{d t}<v_{z}>-\frac{q}{m}\left(E_{z}+<v_{x}>B_{y}-<v_{y}>B_{x}\right)+\xi_{z}\left(T_{\text {eff }, x}, T_{\text {eff }, y}, T_{\text {eff }, z}\right)<v_{z}> \\
& =R\left(v_{z}\right)-\left[<v_{z}>-\xi_{z}\left(T_{\text {eff }, x}, T_{\text {eff }, y}, T_{\text {eff }, z}\right)<v_{z}>\right]
\end{aligned}
$$

for the $z$ direction; similar equations for $x$ and $y$ are obtained by cyclic permutation of the direction labels.

In the multi-temperature theory, the collision frequency for momentum transfer is different for each of the three directions. The definition for direction $u$ is [23]

$$
\begin{aligned}
\xi_{u}\left(T_{e f f, x}, T_{e f f, y}, T_{e f f, z}\right)= & \frac{2 N}{\pi^{3 / 2}}\left(\frac{M}{m+M}\right) \iiint \exp \left(-\gamma_{x}^{2}-\gamma_{y}^{2}-\gamma_{z}^{2}\right) \gamma_{u}^{2} g Q^{(1)} \\
& \times\left(\frac{1}{2} \mu g^{2}\right) d \gamma_{x} d \gamma_{y} d \gamma_{z},
\end{aligned}
$$

where

$$
\frac{1}{2} \mu g^{2}=\gamma_{x}^{2} k_{B} T_{\text {eff }, x}+\gamma_{y}^{2} k_{B} T_{\text {eff }, y}+\gamma_{z}^{2} k_{B} T_{\text {eff }, z} .
$$

Each of the effective temperatures in Equation (A5) is computed from the corresponding ion temperature via an equation similar to Equation (21):

$$
T_{e f f, u}=\frac{m T+M T_{u}}{m+M}
$$

If all three effective temperatures are the same, then $\xi_{u}\left(T_{\text {eff }}, x\right.$, $\left.T_{\text {eff, } y}, T_{\text {eff, } z}\right)$ becomes identical with $\xi\left(T_{\text {eff }}\right)$ as defined by Equation (21).

The multi-temperature moment equation for the effective temperature was given previously [23]. For the $z$ direction, it is

$$
\begin{gathered}
\frac{\partial}{\partial t}\left(\frac{1}{2} k_{B} T_{\text {eff }, z}\right)-\frac{q \mu}{m}\left[E_{z}<v_{z}>+B_{y}<v_{x} v_{z}>-B_{y}<v_{y} v_{z}>\right] \\
+\frac{2 \mu}{M} \xi_{z}\left(T_{\text {eff }, x}, T_{\text {eff }, y}, T_{\text {eff }, z}\right)\left[\frac{1}{2} k_{B}\left(T_{\text {eff }, z}-T\right)+\frac{M \Phi_{z}}{m}\left(\frac{1}{2} k_{B} T_{\text {eff }, z}\right)\right]=\frac{\mu}{m} R\left(\frac{1}{2} m v_{z}^{2}\right) \\
-\left\{<J\left(\frac{1}{2} m v_{z}^{2}\right)>-\frac{2 \mu}{M} \xi_{z}\left(T_{\text {eff }, x}, T_{\text {eff }, y}, T_{\text {eff }, z}\right)\left[\frac{1}{2} k_{B}\left(T_{\text {eff }, z}-T\right)+\frac{M \Phi_{z}}{m}\left(\frac{1}{2} k_{B} T_{\text {eff }, z}\right)\right]\right\} .
\end{gathered}
$$




$$
\begin{aligned}
& \frac{\partial}{\partial t}<v_{x} v_{y}>-\frac{q}{m}\left(E_{x}<v_{y}>+E_{y}<v_{x}>\right)+\frac{q B_{z}}{m^{2}}\left(k_{B} T_{\text {eff }, x}-k_{B} T_{\text {eff }, y}\right) \\
& -\frac{q}{m}\left(B_{x}<v_{x} v_{z}>-B_{y}<v_{y} v_{z}>\right)+\zeta_{x y}\left(T_{\text {eff }, x}, T_{\text {eff }, y}, T_{\text {eff }, z}\right)<v_{x} v_{y}> \\
& \left.=R\left(v_{x} v_{y}\right)-\left[<J\left(v_{x} v_{y}\right)-\zeta_{x y}\left(T_{\text {eff }, x}, T_{\text {eff }, y}, T_{\text {eff }, z}\right)<v_{x} v_{y}>\right)\right],
\end{aligned}
$$

The equations for the other directions can be obtained by cyclic permutations of the $x, y$ and $z$ labels. The new quantities, $\Phi u$ with $u=x, y, z$, are dimensionless ratios of collision integrals that are defined in the appendix of [47]. The terms proportional to the magnetic field components would cancel if Equation (A7) were added to similar equations along the $x$ and $y$ directions, which means that magnetic fields will not affect the overall kinetic energy although, like the $\Phi_{u}$ quantities, they will affect energy partitioning among the various directions in the apparatus.

To complete this model in first approximation, we need moment equations for the cross terms in Equation (A7). It has been shown [23] to be given by Equation (A8), with similar equations for the other directions obtained by cyclic permutation of the labels. The new quantity, $\zeta_{x y}\left(T_{\text {eff, } x}, T_{\text {eff, } y}, T_{\text {eff, }}\right)$, is the collision frequency for correlated velocities along the $x$ and $y$ directions that is defined microscopically in [23].

\section{B. Derivation of Equation (21)}

From Equations (10)-(12), we find that

$$
\frac{1}{2} m v^{2}=k_{B} T_{i}\left[\frac{3}{2}-\psi_{0,0}^{(1)}(v)\right] .
$$

Since $\psi_{0,0}^{(1)}(\boldsymbol{v})=1$, we can use the matrix elements of the collision operator with respect to the Burnett functions [14] along with Equation (B1) to give

$$
\begin{aligned}
<J\left(\frac{1}{2} m v^{2}\right)> & =k_{B} T_{i}\left[\frac{3}{2}<J \psi_{0,0}^{(0)}(\boldsymbol{v})>-<J \psi_{0,0}^{(1)}(\boldsymbol{v})>\right] \\
& =k_{B} T_{i}\left[\frac{3}{2}<\psi_{0,0}^{(0)}(\boldsymbol{v}) J \psi_{0,0}^{(0)}(\boldsymbol{v})>-<\psi_{0,0}^{(0)}(\boldsymbol{v}) J \psi_{0,0}^{(1)}(\boldsymbol{v})>\right] \\
& =k_{B} T_{i}\left[\frac{3}{2} a_{0,0}(0)-a_{1,0}(0)\right] .
\end{aligned}
$$

It has been shown previously [14] that

and

$$
a_{0,0}(0)=0
$$

$$
\begin{aligned}
a_{1,0}(0) & =-8\left(\frac{M}{m+M}\right)^{2} \frac{m\left(T_{i}-T\right)}{M T_{i}}\left(\frac{2 k_{B} T_{e f f}}{\pi \mu}\right)^{\frac{1}{2}} \Omega^{1,1}\left(T_{e f f}\right) \\
& =-\frac{3 M}{m+M} \frac{m\left(T_{i}-T\right)}{M T_{i}} \xi\left(T_{e f f}\right) .
\end{aligned}
$$

Hence

$$
<J\left(\frac{1}{2} m v^{2}\right)>=\frac{3 m}{m+M} k_{B}\left(T_{i}-T\right) \xi\left(T_{e f f}\right)
$$

and Equation (26) follows from Equation (25).

\section{References}

1. Eiceman, G.A., Karpas, Z.: Ion Mobility Spectrometry, 2nd edn. CRC Press, Boca Raton (2005)

2. Revercomb, H.E., Mason, E.A.: Theory of Plasma Chromatograph/ Gaseous Electrophoresis-A Review. Anal. Chem. 47, 970-983 (1975)

3. Gardner, A.M., Gutsmiedl, K.A., Wright, T.G., Breckenridge, W.H., Chapman, C.Y.N., Viehland, L.A.: Theoretical Study of $\mathrm{Al}^{+}-\mathrm{RG}(\mathrm{RG}=$ He-Rn). J. Chem. Phys. 133, 164302 (2010)

4. White, R.D., Ness, K.F., Robson, R.E., Li, B.: Charged-particle transport in electric and magnetic fields crossed at arbitrary angles: Multi-term solution of Boltzmann's equation. Phys. Rev. E 60, 22312249 (1999)

5. Viehland, L.A., Danailov, D.M., Goeringer, D.E.: Moment theory of ion-neutral reactions in traps and similar devices. J. Phys. Chem. 111, 2820-2829 (2007)

6. Viehland, L.A., Chang, Y.: Beyond the Monchick-Mason approximation: The mobility of $\mathrm{Li}^{+}$ions in $\mathrm{H}_{2}$. Mol. Phys. 110, 259-266 (2012)

7. Robson, R.E., Nicoletopoulos, P., Li, B., White, R.D.: Kinetic theoretical and fluid modeling of plasmas and swarms: The Big Picture. Plasma Sources Sci. Technol. 17, 024020 (2008)

8. Boltzmann, L.: Weitere Studien uber das Warmegleichgewicht unter Gasmolekulen Sitzungsber. Kais. Akad. Wiss. Wien Math. Naturwiss. 66, 275-370 (1872). An English translation is available in Brush, S. G. Kinetic Theory, Vol. 2, Irreversible Processes. Pergamon: Oxford, (1966)

9. Cercignani, C.: The Boltzmann Equation and Its Applications. SpringerVerlag, New York (1988)

10. Mason, E.A., McDaniel, E.W.: Transport Properties of Ions in Gases. Wiley, New York (1988)

11. Viehland, L.A., Mason, E.A.: Statistical-mechanical theory of gaseous ion-molecule reactions in an electrostatic field. J. Chem. Phys. 66, 422454 (1977)

12. Weinert, U.: Multi-temperature generalized moment method in Boltzmann transport theory. Phys. Rep. 91, 297-399 (1982)

13. Grad, H.: On the Kinetic theory of rarified gases. Commun. Pure Appl. Math. 2, 325-330 (1949)

14. Viehland, L.A., Mason, E.A.: Gaseous ion mobility in electric fields of arbitrary strength. Ann. Phys. (NY) 91, 499-533 (1975)

15. Viehland, L.A., Mason, E.A.: Gaseous ion mobility and diffusion in electric fields of arbitrary strength. Ann. Phys. (NY) 110, 287-328 (1978)

16. Robson, R.E.: Introductory Transport Theory for Charged Particles in Gases. World Scientific, Singapore (2006)

17. Ender, A.Y., Ender, I.A., Gerasimenko, A.B.: Standard moment method in the problems on ion kinetics in neutral gases. Open Phys. Plasma J 2 , 24-62 (2009)

18. Chapman, S., Cowling, T.G.: The Mathematical Theory of Non-Uniform Gases, 3rd edn. Cambridge University Press, Cambridge (1970) 
19. Maxwell, J.C.: On the Dynamical Theory of Gases. Philos. Trans. R. Soc. London. 157, 49-88 (1867). A reprint is available in Brush, S. G. Kinetic Theory, Vol. 2, Irreversible Processes. Pergamon: Oxford, (1966)

20. Kihara, T.: The Mathematical theory of electrical discharges in gases. B. Velocity distribution of positive ions in a static field. Rev. Mod. Phys. 25, 844-852 (1953)

21. Robson, R.E., White, R.D., Makabe, T.: Charged particle transport in harmonically varying electric fields: Foundations and phenomenology. Ann. Phys. 261, 74-113 (1997)

22. Robson, R.E., White, R.D., Petrović, Z.L.: Colloquium: Physically based fluid modeling of collisionally dominated low-temperature plasmas. Rev. Mod. Phys. 77, 1303-1320 (2005)

23. Viehland, L.A., Goeringer, D.E.: Moment theory of ion motion in traps and similar devices. I. General theories. J. Phys. B. 38, 3987-4009 (2005)

24. Lin, S.L., Viehland, L.A., Mason, E.A.: Three-temperature theory of gaseous ion transport. Chem. Phys. 37, 411-424 (1979)

25. Viehland, L.A., Lin, S.L.: Application of the three-temperature theory of gaseous ion transport. Chem. Phys. 43, 135-144 (1979)

26. Numerous tables of ion-neutral interaction potentials and gaseous ion transport data can be obtained from www.icecat.laplace.univ-tlse.fi

27. Hershey, A.V.A.: Theory for the mobility of ions of high velocity. Phys. Rev. 56, 916-922 (1939)

28. Wannier, G.H.: Motion of gaseous ions in strong electric fields. Bell Syst. Technol. J. 32, 170-254 (1953)

29. Hickling, H.L., Viehland, L.A., Shepherd, D.T., Soldan, P., Lee, E.P.F., Wright, T.G.: Spectroscopy of $\mathrm{M}^{+}-\mathrm{Rg}$ and transport coefficients of $\mathrm{M}^{+}$ in $\mathrm{Rg}(\mathrm{M}=\mathrm{Rb}-\mathrm{Fr}, \mathrm{Rg}=\mathrm{He}-\mathrm{Rn})$. Phys. Chem. Chem. Phys. 6, 4233-4239 (2004)

30. Buchachenko, A.A., Tscherbul, T.V., Klos, J., Szczesniak, M.M., Chalasinski, G., Webb, R., Viehland, L.A.: Interaction potentials of the $\mathrm{Rg}-\mathrm{I}$ anions, neutrals, and cations ( $\mathrm{Rg}=\mathrm{He}, \mathrm{Ne}, \mathrm{Ar})$. J. Chem. Phys. 122, 194311 (2005)

31. Viehland, L.A., Chang, Y.: Transport cross sections for collisions between particles. Comput. Phys. Commun. 181, 1687-1696 (2010)

32. Yousef, A., Shrestha, S., Viehland, L.A., Lee, E.P.F., Gray, B.R., Ayles, V.L., Wright, T.G., Breckenridge, W.H.: Interaction potentials and transport properties of coinage metal cations in rare gases. J. Chem. Phys. 127, 154309 (2007)

33. Langevin, P.: Sur Une Formule Fundamentale de Theorie Cinetique. Ann. Chim. Phys. 5, 245-288 (1905). An English translation is available in McDaniel, E. W. Collision Phenomena in Ionized Gases. Wiley: New York, (1984)

34. Hassé, H.R., Cook, W.R.: The calculation of the mobility of monomolecular ions. Philos. Mag. 12, 554-566 (1931)
35. Tyndall, A.M.: Mobility of Positive Ions. Cambridge Physical Tracts. Cambridge Univ. Press, Cambridge (1938)

36. Mason, E.A., Schamp Jr., H.W.: Mobility of gaseous ions in weak electric fields. Ann. Phys. 4, 233-270 (1958)

37. Viehland, L.A.: Zero-field mobilities in helium: Highly accurate values for use in ion mobility spectrometry. Int. J. Ion Mobil. Spectrom. 15, 21-29 (2012)

38. Viehland, L.A., Mason, E.A., Whealton, J.H.: Kinetic theory of ion cyclotron resonance collision broadening. J. Chem. Phys. 62, 47154726 (1975)

39. Viehland, L.A., Mason, E.A.: Ion-molecule rate coefficients from collision-dominated ion cyclotron resonance. Int. J. Mass Spectrom. Ion Phys. 21, 43-56 (1976)

40. Buryakov, I.A., Krylov, E.V., Nazarov, E.G., Rasulev, U.K.: A new method of separation of multi-atomic ions by mobility at atmospheric pressure using a high-frequency amplitude asymmetric strong electric field. Int. J. Mass Spectrom. Ion Process. 128, 143-148 (1993)

41. Guevremont, R., Purves, R.W.: High field asymmetric waveform ion mobility spectrometry-mass spectrometry: An investigation of leucine enkephalin ions produced by electrospray ionization. J. Am. Soc. Mass Spectrom. 10, 492-501 (1999)

42. Papanastasiou, D., Wollnik, H., Rico, G., Tadjimukhamedov, F., Mueller, W., Eiceman, G.A.: Differential mobility separation of ions using a rectangular asymmetric waveform. J. Phys. Chem. A 112, 3638-3645 (2008)

43. Lin, S.L., Viehland, L.A., Mason, E.A., Whealton, J.H., Bardsley, J.N.: Velocity and energy relaxation of ions in drift tubes. J. Phys. B Atomic Mol. Opt. Phys. 10, 3567-3575 (1977)

44. Shvartsburg, A.A., Mashkevich, S.V., Smith, R.D.: Feasibility of higher-order differential ion mobility separations using new asymmetric waveforms. J. Phys. Chem. A 110, 2663-2673 (2006)

45. Robson, R.E., Kumar, K.: Mobility and diffusion. II. Dependence upon experimental variables and interaction potential for alkali ions in rare gases. Aust. J. Phys. 26, 187-201 (1973)

46. Goeringer, D.E., Viehland, L.A.: Moment theory of ion motion in traps and similar devices. III. Two-temperature treatment of quadrupole ion traps. J. Phys. B Atomic Mol. Opt. Phys. 38, 4027-4044 (2005)

47. Viehland, L.A., Danailov, D.M., Goeringer, D.E.: Moment theory of ion motion in traps and similar devices. V. Multi-temperature treatment of quadrupole ion traps. J. Phys. B Atomic Mol. Opt. Phys. 39, 4015-4035 (2006)

48. Robson, R.E.: Physics of reacting particle swarms in gases. J. Chem. Phys. 85, 4486-4501 (1986)

49. Li, B., White, R.D., Robson, R.E., Ness, K.F.: Transport coefficients in crossed E and B fields: Empirical relations and nonconservative collisional effects. Ann. Phys. 292, 179-198 (2001) 